\title{
FINDING THE PROBABILISTICALLY-TEMPORAL CHARACTERISTICS OF MARKOV G-NETWORK WITH BATCH REMOVAL OF POSITIVE CUSTOMERS
}

\author{
Mikhail Matalytski $^{1}$, Victor Naumenko ${ }^{2}$, Dmitry Kopats ${ }^{2}$ \\ ${ }^{1}$ Institute of Mathematics, Czestochowa University of Technology \\ Czestochowa, Poland \\ ${ }^{2}$ Faculty of Mathematics and Computer Science, Grodno State University \\ Grodno, Belarus \\ m.matalytski@gmail.com,victornn86@gmail.com
}

Received: 04 May 2016; accepted: 11 July 2016

\begin{abstract}
The investigation of a Markov queueing network with positive and negative customers and positive customers batch removal has been carried out in the article. The purpose of the research is analysis of such a network at the non-stationary regime, finding the time-dependent state probabilities and mean number of customers. In the first part of this article, a description of the G-network operation is provided with one-line queueing systems. When a negative customer arrives to the system, the count of positive customers is reduced by a random value, which is set by some probability distribution. Then for the non-stationary state probabilities a Kolmogorov system was derived of difference-differential equations. A technique for finding the state probabilities and the mean number of customers of the investigated network, based on the use of an apparatus of multidimensional generating functions has been proposed. The theorem about the expression for the generating function has been given. A model example has been calculated.
\end{abstract}

Keywords: G-network, positive and negative customers, customers batch removal, nonstationary regime, generation function, state probabilities

\section{Introduction}

G-networks or queueing networks with positive and negative customers was introduced by Gelenbe [1]. Such networks with batch removal of positive customers are used in the development of computer systems and networks models, in which defective problems (negative customers) destroy tasks or user jobs (positive customers). Task handlers are the processes running on servers; customers - user jobs for processing [2].

Let's consider an open G-queueing network with $n$ single-line queueing systems (QS). To the $S_{i}$ system external environment (QS $S_{0}$ ) a simple flow of customers arrives with the rate $\lambda_{0 i}^{+}$and a simple flow of negative customers with the rate $\lambda_{0 i}^{-}, i=\overline{1, n}$. All customer flows that arrive to the network are independent. 
The service durations of positive customers in $i$-th QS distributed exponentially with the rate $\mu_{i}, i=\overline{1, n}$.

In the articles [3-5], a G-network at a transient (non-stationary) regime has been investigated. At that negative customer, arrivining to the system, where there were positive customers, immediately destroy one of them, and then immediately left the network, not having received service in the QS. In the article [6] was conducted analysis at non-stationary regime of G-network with signals, and in the article [7] G-network with signals with random delay.

In this paper, we describe in more detail the other behaviors of negative customers, which arrive to the network systems. Let at time $t$ in the system $S_{i}$ there are $k_{i} \geq B_{i}$ positive customers, where $B_{i}$ - integer random value. Negative customer arriving to the some system of the network instantly destroys (removes from the network) destroyed immediately $B_{i}$ of positive customers. If $k_{i}<B_{i}$, the system $S_{i}$ completely is devastated (all positive customers, which are in this QS at a given time are immediately destroyed). Thus, random value $B_{i}$, which effectively determines the maximum size of the destruction batch of customers in QS $S_{i}$, is subject to an arbitrary discrete distribution law:

$$
P\left\{B_{i}=m\right\}=\pi_{i m}, m \geq 1 .
$$

Gelenbe studied such behavior of negative customers considering signals in the paper [8], but only at stationary regime.

As previously explained, in each network system only positive customers are serviced. A positive customer serviced in the QS $S_{i}$, with probability $p_{i j}^{+}$sent to the QS $S_{j}$ as a positive customer, with a probability $p_{i j}^{-}$- as a negative customer, and with probability $p_{i 0}=1-\sum_{j=1}^{n}\left(p_{i j}^{+}+p_{i j}^{-}\right)$leaving from the network to the external environment (QS $\left.S_{0}\right), i, j=\overline{1, n}$.

The state of the network at time $t$ meaning the vector

$$
k(t)=(k, t)=\left(\left(k_{1}, t\right),\left(k_{2}, t\right), \ldots,\left(k_{n}, t\right)\right),
$$

which forms a Markov random process with a countable number of states, where state $\left(k_{i}, t\right)$ means that at time $t$ in QS $S_{i}$ there are $k_{i}$ of positive customers, $i=\overline{1, n}$.

\section{The system of the difference-differential Kolmogorov equations for the network state probabilities}

We introduce some notations. Let's $P(k, t)$ - probability of the network state $k$ at time $t ; u(x)$ - Heaviside function, $u(x)=\left\{\begin{array}{l}1, x>0 ; \\ 0, x \leq 0 .\end{array} ; I_{i}\right.$ - a vector of dimension 
$n$, consisting of zeros, except for the component with number of $i$, which is equal to $1, i=\overline{1, n}$. Let's allows

$$
\begin{gathered}
k_{i}^{+}=k+I_{i}=\left(k_{1}, k_{2}, \ldots, k_{i}+1, \ldots, k_{n}\right), \\
k_{i}^{+m}=k+m I_{i}=\left(k_{1}, k_{2}, \ldots, k_{i}+m, \ldots, k_{n}\right), m \geq 1, \\
k_{i}^{-}=k-I_{i}=\left(k_{1}, k_{2}, \ldots, k_{i}-1, \ldots, k_{n}\right), \\
k_{i j}^{+-}=k+I_{i}-I_{j}=\left(k_{1}, k_{2}, \ldots, k_{i}+1, \ldots, k_{j}-1, \ldots, k_{n}\right), \\
k_{i j}^{++m}=k+I_{i}+m I_{j}=\left(k_{1}, k_{2}, \ldots, k_{i}+1, \ldots, k_{j}+m, \ldots, k_{n}\right), m \geq 1 .
\end{gathered}
$$

There are following transitions of a random process $k(t)$ to the state $(k, t)$ during time $\Delta t$ :

1) from the state $\left(k_{i}^{-}, t\right)$, in this case, during time $\Delta t$ a positive customer arriving to the QS $S_{i}$ with probability $\lambda_{0 i}^{+} u\left(k_{i}(t)\right) \Delta t+o(\Delta t)$;

2) from the state $\left(k_{i}^{+}, t\right)$, wherein the positive customer leaves the network or in an external environment passes into QS $S_{j}$ as a negative customer, if it had no customers; the probability of such an event equals $\left(\mu_{i} p_{i 0}+\mu_{i} p_{i j}^{-}\left(1-u\left(k_{j}(t)\right)\right)\right) \Delta t+o(\Delta t)$

3) from the states $\left(k_{i}^{+m}, t\right)$, in this case a negative customer arrives to the QS $S_{i}$ and destroys a random batch of positive customers; the probability of such event is equal to $\lambda_{0 i}^{-}\left(1-u\left(k_{i}(t)\right)\right) \sum_{m=1}^{\infty} \pi_{i m} \Delta t+o(\Delta t)$;

4) from the state $\left(k_{i}^{+-}, t\right)$, in this case, after the servicing of a positive customer in the QS $S_{i}$ it goes to the QS $S_{j}$; the probability of such an event is equal to $\mu_{i} p_{i j}^{+} u\left(k_{j}(t)\right) \Delta t+o(\Delta t)$

5) from the states in this case, after the servicing of a positive customer in the QS $S_{i}$ it goes to the QS $S_{j}$ as a negative customer, which destroys in it random batch of positive customers; the probability of such an event is equal to $\mu_{i} p_{i j}^{-}\left(1-u\left(k_{j}(t)\right)\right) \sum_{m=1}^{\infty} \pi_{i m} \Delta t+o(\Delta t)$

6) from the state $(k, t)$, while in each QS $S_{i}, i=\overline{1, n}$, there are no positive customers nor any negative customers arriving, and which during $\Delta t$ was not to serviced by any customer; the probability of such event is equal to $1-\sum_{i=1}^{n}\left[\lambda_{0 i}^{+}+\lambda_{0 i}^{-}+\mu_{i}\right] \Delta t+o(\Delta t), i=\overline{1, n} ;$

7) of the remaining states with a probability $o(\Delta t)$. 
Then, using the formula of total probability, we shall have

$$
\begin{aligned}
P(k, t+\Delta t)=\sum_{i=1}^{n}\left[\left(\lambda_{0 i}^{+} \Delta t+o(\Delta t)\right) P\left(k_{i}^{-}, t\right) u\left(k_{i}(t)\right)+\right. & \\
& +\lambda_{0 i}^{-} \sum_{m=1}^{\infty}\left(\pi_{i m} \Delta t+o(\Delta t)\right) P\left(k_{i}^{+m}, t\right)+ \\
+ & \lambda_{0 i}^{-} \sum_{m=1}^{\infty} \pi_{i m} \sum_{r=0}^{m-1} P\left(k_{i}^{+r}, t\right)\left(1-u\left(k_{i}(t)\right)\right) \Delta t+o(\Delta t)+ \\
+ & \sum_{j=1}^{n}\left\{\left(\mu_{i} p_{i j}^{+} \Delta t+o(\Delta t)\right) P\left(k_{i j}^{+-}, t\right) u\left(k_{j}(t)\right)+\right. \\
& +\sum_{m=1}^{\infty} \pi_{i m}\left(\mu_{i} p_{i j}^{-} \Delta t+o(\Delta t)\right) P\left(k_{i j}^{++m}, t\right)+ \\
+ & \sum_{m=1}^{\infty} \pi_{i m} \sum_{r=0}^{m-1} P\left(k_{i j}^{++r}, t\right)\left(1-u\left(k_{j}(t)\right)\right) \Delta t+o(\Delta t)+ \\
& \left.+\left(\mu_{i} p_{i j}^{-} \Delta t+o(\Delta t)\right) P\left(k_{i}^{+}, t\right)\left(1-u\left(k_{j}(t)\right)\right)\right\}+ \\
+\left(\mu_{i} p_{i 0} \Delta t\right. & \left.+o(\Delta t)) P\left(k_{i}^{+}, t\right)+\left(1-\left(\lambda_{0 i}^{+}+\lambda_{0 i}^{-}+\mu_{i}\right) \Delta t+o(\Delta t)\right) P(k, t)\right] .
\end{aligned}
$$

Dividing both sides of this relation by $\Delta t$ and passing to the limit $\Delta t \rightarrow 0$, we obtain that the non-stationary states probabilities of the considered network satisfy the following system of difference-differential equations

$$
\begin{gathered}
\frac{d P(k, t)}{d t}=-\sum_{i=1}^{n}\left[\lambda_{0 i}^{+}+\lambda_{0 i}^{-}+\mu_{i}\right] P(k, t)+\sum_{i=1}^{n} \lambda_{0 i}^{+} u\left(k_{i}(t)\right) P\left(k_{i}^{-}, t\right)+ \\
+\sum_{i=1}^{n}\left[\mu_{i}\left(p_{i 0}+\sum_{j=1}^{n} p_{i j}^{-}\left(1-u\left(k_{j}(t)\right)\right)\right] P\left(k_{i}^{+}, t\right)+\right. \\
+\sum_{i=1}^{n} \lambda_{0 i}^{-}\left\{\sum_{m=1}^{\infty} \pi_{i m}\left[P\left(k_{i}^{+m}, t\right)+\left(1-u\left(k_{i}(t)\right)\right) \sum_{r=0}^{m-1} P\left(k_{i}^{+r}, t\right)\right]\right\}+ \\
+\sum_{i, j=1}^{n}\left\{\mu_{i} p_{i j}^{+} u\left(k_{j}(t)\right) P\left(k_{i j}^{+-}, t\right)+\sum_{m=1}^{\infty} \pi_{i m}\left[\mu_{i} p_{i j}^{-} P\left(k_{i j}^{++m}, t\right)+\left(1-u\left(k_{j}(t)\right)\right) \sum_{r=0}^{m-1} P\left(k_{i j}^{++r}, t\right)\right]\right\} .
\end{gathered}
$$

Suppose that all systems of the network operating in a heavy traffic regime, i.e. $k_{i}(t)>0, \forall t>0, i=\overline{1, n}$. Taking this assumption into account, the system (5) takes the form 


$$
\begin{gathered}
\frac{d P(k, t)}{d t}=-\sum_{i=1}^{n}\left[\lambda_{0 i}^{+}+\lambda_{0 i}^{-}+\mu_{i}\right] P(k, t)+ \\
\sum_{i=1}^{n} \lambda_{0 i}^{+} P\left(k_{i}^{-}, t\right)+\sum_{i=1}^{n} \mu_{i} p_{i 0} P\left(k_{i}^{+}, t\right)+\sum_{i=1}^{n} \lambda_{0 i}^{-} \sum_{m=1}^{\infty} \pi_{i m} P\left(k_{i}^{+m}, t\right)+ \\
+\sum_{i, j=1}^{n}\left[\mu_{i} p_{i j}^{+} P\left(k_{i j}^{+-}, t\right)+\sum_{m=1}^{\infty} \pi_{i m} \mu_{i} p_{i j}^{-} P\left(k_{i j}^{++m}, t\right)\right] .
\end{gathered}
$$

\section{Finding network state probabilities using the generating function}

Denote by $\Psi_{n}(z, t)$, where $z=\left(z_{1}, z_{2}, \ldots, z_{n}\right),|z|<1, n$-dimensional generating function:

$$
\Psi_{n}(z, t)=\sum_{k_{1}=0 k_{2}=0}^{\infty} \sum_{k_{n}=0}^{\infty} \ldots \sum^{\infty} P\left(k_{1}, . ., k_{n}, t\right) z_{1}^{k_{1}} z_{2}^{k_{2}} \cdot \ldots \cdot z_{n}^{k_{n}}=\sum_{k_{1}=0}^{\infty} \ldots \sum_{k_{n}=0}^{\infty} P(k, t) \prod_{i=1}^{n} z_{i}^{k_{i}}
$$

Multiplied (6) on $\prod_{l=1}^{n} z_{l}^{k_{l}}$ and adding together all possible values $k_{l}$ from 0 to $+\infty, l=\overline{1, n}$, obtain:

$$
\begin{gathered}
\sum_{k_{1}=0}^{\infty} \ldots \sum_{k_{\mathrm{n}}=0}^{\infty} \frac{d P(k, t)}{d t} \prod_{l=1}^{n} z_{l}^{k_{l}}=-\sum_{i=1}^{n}\left(\lambda_{0 i}^{+}+\lambda_{0 i}^{-}+\mu_{i}\right) \sum_{k_{1}=0}^{\infty} \ldots \sum_{k_{n}=0}^{\infty} P(k, t) \prod_{l=1}^{n} z_{l}^{k_{l}}+ \\
+\sum_{i=1}^{n} \lambda_{0 i}^{+} \sum_{k_{1}=0}^{\infty} \ldots \sum_{k_{n}=0}^{\infty} P\left(k_{i}^{-}, t\right) \prod_{l=1}^{n} z_{l}^{k_{l}}+\sum_{i=1}^{n} \mu_{i} p_{i 0} \sum_{k_{1}=0}^{\infty} \ldots \sum_{k_{n}=0}^{\infty} P\left(k_{i}^{+}, t\right) \prod_{l=1}^{n} z_{l}^{k_{l}}+ \\
+\sum_{i=1}^{n} \lambda_{0 i}^{-} \sum_{k_{1}=0}^{\infty} \ldots \sum_{k_{n}=0}^{\infty} \sum_{m=1}^{\infty} \pi_{i m} P\left(k_{i}^{+m}, t\right) \prod_{l=1}^{n} z_{l}^{k_{l}}+\sum_{i, j=1}^{n} \mu_{i} p_{i j}^{+} \sum_{k_{1}=0}^{\infty} \ldots \sum_{k_{\mathrm{n}}=0}^{\infty} P\left(k_{i j}^{+-}, t\right) \prod_{l=1}^{n} z_{l}^{k_{l}}+ \\
+\sum_{i, j=1}^{n} \sum_{m=1}^{\infty} \pi_{i m} \mu_{i} p_{i j}^{-} \sum_{k_{1}=0}^{\infty} \ldots \sum_{k_{\mathrm{n}}=0}^{\infty} P\left(k_{i j}^{++m}, t\right) \prod_{l=1}^{n} z_{l}^{k_{l}} .
\end{gathered}
$$

Consider some sums, contained on the right side of (8). Let's allows

$$
\sum_{1}(z, t)=\sum_{i=1}^{n} \lambda_{0 i}^{+} \sum_{k_{1}=0}^{\infty} \ldots \sum_{k_{n}=0}^{\infty} P\left(k_{i}^{-}, t\right) \prod_{l=1}^{n} z_{l}^{k_{l}}
$$

then we can show that

$$
\sum_{1}(z, t)=\sum_{i=1}^{n} \lambda_{0 i}^{+} z_{i} \sum_{\substack{k_{j}=0 \\ j=1, n}}^{\infty} P(k, t) \prod_{l=1}^{n} z_{l}^{k_{l}}=\sum_{i=1}^{n} \lambda_{0 i}^{+} z_{i} \Psi_{n}(z, t) .
$$


For the sum of $\sum_{2}(z, t)=\sum_{i=1}^{n} \mu_{i} p_{i 0} \sum_{k_{1}=0}^{\infty} \ldots \sum_{k_{n}=0}^{\infty} P\left(k_{i}^{+}, t\right) \prod_{l=1}^{n} z_{l}^{k_{l}}$ we have:

$$
\sum_{2}(z, t)=\sum_{i=1}^{n} \frac{\mu_{i} p_{i 0}}{z_{i}} \Psi_{n}(z, t)-\sum_{i=1}^{n} \frac{\mu_{i} p_{i 0}}{z_{i}} \sum_{\substack{k_{j}=0 \\ j=1, n, j \neq i}}^{\infty} P\left(k_{1}, \ldots, k_{i-1}, 0, k_{i+1} . ., k_{n}, t\right) \prod_{\substack{l=1 \\ l \neq i}}^{n} z_{l}^{k_{l}}
$$

For the sum of $\sum_{3}(z, t)=\sum_{i=1}^{n} \lambda_{0 i}^{-} \sum_{k_{1}=0}^{\infty} \ldots \sum_{k_{n}=0}^{\infty} \sum_{m=1}^{\infty} \pi_{i m} P\left(k_{i}^{+m}, t\right) \prod_{l=1}^{n} z_{l}^{k_{l}}$ we will have:

$\sum_{3}(z, t)=\sum_{i=1}^{n} \sum_{m=1}^{\infty} \pi_{i m} \frac{\lambda_{0 i}^{-}}{z_{i}^{m}} \Psi_{n}(z, t)-\sum_{i=1}^{n} \sum_{m=1}^{\infty} \pi_{i m} \frac{\lambda_{0 i}^{-}}{z_{i}^{m}} \sum_{\substack{k_{j}=0 \\ j=1, n, j \neq i}}^{\infty} P\left(k_{1}, \ldots, k_{i-1}, 0, k_{i+1} . ., k_{n}, t\right) \prod_{\substack{l=1 \\ l \neq i}}^{n} z_{l}^{k_{l}}$.

For the sum of $\sum_{4}(z, t)=\sum_{i, j=1}^{n} \mu_{i} p_{i j}^{+} \sum_{k_{1}=0}^{\infty} \ldots \sum_{k_{n}=0}^{\infty} P\left(k+I_{i}-I_{j}, t\right) \prod_{l=1}^{n} z_{l}^{k_{l}}$ we obtain:

$$
\begin{gathered}
\sum_{4}(z, t)=\sum_{i, j=1}^{n} \mu_{i} p_{i j}^{+} \frac{z_{j}}{z_{i}} \Psi_{n}(z, t)- \\
-\sum_{i, j=1}^{n} \mu_{i} p_{i j}^{+} \frac{z_{j}}{z_{i}} \sum_{\substack{k_{m}=0 \\
m=1, n, m \neq j}}^{\infty} P\left(k_{1}, \ldots, k_{i-1}, 0, k_{i+1}, \ldots, k_{n}, t\right) \prod_{\substack{l=1 \\
l \neq i}}^{n} z_{l}^{k_{l}} .
\end{gathered}
$$

And finally, for the last sum

$$
\sum_{5}(z, t)=\sum_{i, j=1}^{n} \sum_{m=1}^{\infty} \pi_{i m} \mu_{i} p_{i j}^{-} \sum_{k_{1}=0}^{\infty} \ldots \sum_{k_{\mathrm{n}}=0}^{\infty} P\left(k_{i j}^{++m}, t\right) \prod_{l=1}^{n} z_{l}^{k_{l}}
$$

we shall have:

$$
\begin{gathered}
\sum_{5}(z, t)=\sum_{i, j=1 m=1}^{n} \sum_{i m}^{\infty} \mu_{i} p_{i j}^{-} \frac{1}{z_{i} z_{j}^{m}} \Psi_{n}(z, t)- \\
-\sum_{i, j=1}^{n} \sum_{m=1}^{\infty} \pi_{i m} \mu_{i} p_{i j}^{-} \frac{1}{z_{i} z_{j}^{m}} \sum_{\substack{k_{s}=0 \\
s=1, n, s \neq j}}^{\infty} P\left(k_{1}, \ldots, k_{i-1}, 0, k_{i+1}, \ldots, k_{n}, t\right) \prod_{\substack{l=1 \\
l \neq i}}^{n} z_{l}^{k_{l}} .
\end{gathered}
$$

Therefore, the generating function is a fairly ordinary inhomogeneous linear ordinary differential equation 


$$
\begin{aligned}
& \frac{d \Psi_{n}(z, t)}{d t}=-\left[\sum_{i=1}^{n}\left(\lambda_{0 i}^{+}+\lambda_{0 i}^{-}+\mu_{i}\right)-\sum_{i=1}^{n} \lambda_{0 i}^{+} z_{i}-\sum_{i=1}^{n} \frac{\mu_{i} p_{i 0}}{z_{i}}-\sum_{i=1}^{n} \sum_{m=1}^{\infty} \pi_{i m} \frac{\lambda_{0 i}^{-}}{z_{i}^{m}}-\right. \\
&\left.-\sum_{i, j=1}^{n} \mu_{i} p_{i j}^{+} \frac{z_{j}}{z_{i}}-\sum_{i, j=1}^{n} \sum_{m=1}^{\infty} \pi_{i m} \mu_{i} p_{i j}^{-} \frac{1}{z_{i} z_{j}^{m}}\right] \Psi_{n}(z, t)- \\
&-\sum_{i=1}^{n} \frac{\mu_{i} p_{i 0}}{z_{i}} \sum_{\substack{k_{j}=0 \\
j=1, n, j \neq i}}^{\infty} P\left(k_{1}, \ldots, k_{i-1}, 0, k_{i+1} . ., k_{n}, t\right) \prod_{\substack{l=1 \\
l \neq i}}^{n} z_{l}^{k_{l}}- \\
&-\sum_{i=1}^{n} \sum_{m=1}^{\infty} \pi_{i m} \frac{\lambda_{0 i}^{-}}{z_{i}^{m}} \sum_{\substack{k_{j}=0 \\
j=1, n, j \neq i}}^{\infty} P\left(k_{1}, \ldots, k_{i-1}, 0, k_{i+1} . ., k_{n}, t\right) \prod_{\substack{l=1 \\
l \neq i}}^{n} z_{l}^{k_{l}}- \\
&- \sum_{i, j=1}^{n} \mu_{i} p_{i j}^{+} \frac{z_{j}}{z_{i}} \sum_{\substack{k_{m}=0 \\
m=1, n, m \neq j}}^{\infty} P\left(k_{1}, \ldots, k_{i-1}, 0, k_{i+1}, \ldots, k_{n}, t\right) \prod_{\substack{l=1 \\
l \neq i}}^{n} z_{l}^{k_{l}}- \\
&-\sum_{i, j=1}^{n} \sum_{m=1}^{\infty} \pi_{i m} \mu_{i} p_{i j}^{-} \frac{1}{z_{i} z_{j}^{m}} \sum_{\substack{k_{s}=0 \\
s=1, n, s \neq j}}^{\infty} P\left(k_{1}, \ldots, k_{i-1}, 0, k_{i+1}, \ldots, k_{n}, t\right) \prod_{l=1}^{n} z_{l}^{k_{l}} .
\end{aligned}
$$

Since all of the whole QS network operate under high load conditions, the last two expressions in the form of the sums in equation (9) will be zero, and it becomes homogeneous:

$$
\begin{aligned}
\frac{d \Psi_{n}(z, t)}{d t}= & -\left[\sum_{i=1}^{n}\left(\lambda_{0 i}^{+}+\lambda_{0 i}^{-}+\mu_{i}\right)-\sum_{i=1}^{n} \lambda_{0 i}^{+} z_{i}-\sum_{i=1}^{n} \frac{\mu_{i} p_{i 0}}{z_{i}}-\sum_{i=1}^{n} \sum_{m=1}^{\infty} \pi_{i m} \frac{\lambda_{0 i}^{-}}{z_{i}^{m}}-\right. \\
& \left.-\sum_{i, j=1}^{n} \mu_{i} p_{i j}^{+} \frac{z_{j}}{z_{i}}-\sum_{i, j=1 m=1}^{n} \sum_{i m}^{\infty} \pi_{i m} \mu_{i} p_{i j}^{-} \frac{1}{z_{i} z_{j}^{m}}\right] \Psi_{n}(z, t) .
\end{aligned}
$$

Its general solution has the form

$$
\begin{aligned}
\Psi_{n}(z, t)=C_{n} \exp & \left\{-\sum_{i=1}^{n}\left[\lambda_{0 i}^{+}+\lambda_{0 i}^{-}+\mu_{i}-\lambda_{0 i}^{+} z_{i}-\frac{1}{z_{i}}\left(\mu_{i} p_{i 0}+\sum_{m=1}^{\infty} \frac{\pi_{i m} \lambda_{0 i}^{-}}{z_{i}^{m-1}}-\right.\right.\right. \\
& \left.\left.\left.-\mu_{i} \sum_{j=1}^{n} p_{i j}^{+} z_{j}-\mu_{i} \sum_{j=1}^{n} \sum_{m=1}^{\infty} \pi_{i m} \mu_{i} p_{i j}^{-} \frac{1}{z_{j}^{m}}\right)\right] t\right\} .
\end{aligned}
$$

We assume that at the initial moment of time, the network is in state $\left(\alpha_{1}, \alpha_{2}, \ldots, \alpha_{n}, 0\right), \quad \alpha_{i}>0, \quad P\left(\alpha_{1}, \alpha_{2}, \ldots, \alpha_{n}, 0\right)=1, \quad P\left(k_{1}, k_{2}, \ldots, k_{n}, 0\right)=0, \quad \forall \alpha_{i} \neq k_{i}$, $i=\overline{1, n}$. Then the initial condition for the last equation (10) will be 
$\Psi_{n}(z, 0)=P\left(\alpha_{1}, \alpha_{2}, \ldots, \alpha_{n}, 0\right) \prod_{l=1}^{n} z_{l}^{\alpha_{l}}=\prod_{l=1}^{n} z_{l}^{\alpha_{l}}$. Using it, we obtain $C_{n}=1$. Thus, the expression for the generating function $\Psi_{n}(z, t)$ has the form

$$
\begin{aligned}
\Psi_{n}(z, t) & =a_{0}(t) \exp \left\{\sum_{i=1}^{n} \lambda_{0 i}^{+} z_{i} t\right\} \exp \left\{\sum_{i=1}^{n} \frac{\mu_{i} p_{i 0}}{z_{i}} t\right\} \exp \left\{\sum_{i=1}^{n} \sum_{m=1}^{\infty} \pi_{i m} \frac{\lambda_{0 i}^{-}}{z_{i}^{m}} t\right\} \times \\
& \times \exp \left\{\sum_{i, j=1}^{n} \mu_{i} p_{i j}^{+} \frac{z_{j}}{z_{i}} t\right\} \exp \left\{\sum_{i, j=1}^{n} \sum_{m=1}^{\infty} \pi_{i m} \mu_{i} p_{i j}^{-} \frac{1}{z_{i} z_{j}^{m}} t\right\} \prod_{l=1}^{n} z_{l}^{\alpha_{l}},
\end{aligned}
$$

where

$$
a_{0}(t)=\exp \left\{-\sum_{i=1}^{n}\left(\lambda_{0 i}^{+}+\lambda_{0 i}^{-}+\mu_{i}\right) t\right\}
$$

To find the probability of the network states, we transform (11) to a convenient form by expanding its member exhibitors in a Maclaurin series. We can show that the statement is true.

Theorem. The expression for the generating function can be represented in the form

$$
\begin{gathered}
\Psi_{n}(z, t)=a_{0}(t) \sum_{l_{1}=0}^{\infty} \ldots \sum_{l_{n}=0}^{\infty} \sum_{q_{1}=0}^{\infty} \ldots \sum_{q_{n}=0}^{\infty} \sum_{\eta_{1}=0}^{\infty} \ldots \sum_{r_{n}=0}^{\infty} \sum_{u_{1}=0}^{\infty} \ldots \sum_{u_{n}=0}^{\infty} \sum_{w_{1}=0}^{\infty} \ldots \sum_{w_{n}=0}^{\infty} t_{i=1}^{n}\left(i_{i}+q_{i}+r_{i}+u_{i}+w_{i}\right) \\
\times \prod_{i=1}^{n}\left[\frac{\left(\lambda_{0 i}^{+}\right)^{\gamma_{i}}\left(\mu_{i} p_{i 0}\right)^{q_{i}}\left(\lambda_{0 i}^{-} \prod_{m=1}^{\infty} \pi_{i m}\right)^{r_{i}} \mu_{i}^{u_{i}+w_{i}}\left(\prod_{j=1}^{n} p_{i j}^{+}\right)^{u_{i}}\left(\prod_{j=1}^{n} \prod_{m=1}^{\infty} \pi_{i m} p_{i j}^{-}\right)^{w_{i}}}{l_{i} ! q_{i} ! r_{i} ! u_{i} ! w_{i} !} \times\right. \\
\left.\times z_{i}^{\alpha_{i}+l_{i}-q_{i}-m r_{i}-u_{i}-U-m w_{i}-m W}\right],
\end{gathered}
$$

where $U=\sum_{i=1}^{n} u_{i}, W=\sum_{i=1}^{n} w_{i}$.

The probability $P\left(k_{1}, k_{2}, \ldots, k_{n}, t\right)$ can be found as the coefficient of $z_{1}^{k_{1}} z_{2}^{k_{2}} \ldots z_{n}^{k_{n}}$ in the expansion of function $\Psi_{n}(z, t)$ in multiple series (12), and the mean number of customers in the system $S_{i}$ in the form $N_{i}(t)=\left.\frac{\partial \Psi_{n}(z, t)}{\partial z_{i}}\right|_{z=(1,1, \ldots, 1)}$. 


\section{Model example}

In this example the count of QS $n=5$ lets arriving probabilities of customers to $i$-th QS be respectively equal $p_{01}^{+}=0.3, \quad p_{02}^{+}=0.1, \quad p_{03}^{+}=p_{04}^{+}=p_{05}^{+}=0.2$, $p_{01}^{-}=p_{02}^{-}=p_{03}^{-}=p_{04}^{-}=p_{05}^{-}=0.2$, and $\sum_{i=1}^{5} p_{0 i}^{+}=1, \sum_{i=1}^{5} p_{0 i}^{-}=1$.

Consider the time interval $[0 ; T]$. As a time unit we take 1 hour, then $T=24 \mathrm{~h}$. Let the arriving rates of positive and negative customers be respectively equal $\lambda^{+}=100$ and $\lambda^{-}=90$. Let's arriving rates of positive and negative customers to each QS $\lambda_{0 i}^{+}$and $\lambda_{0 i}^{-}$, taking into account that $\lambda_{0 i}^{+}=\lambda^{+} p_{0 i}^{+}, \lambda_{0 i}^{-}=\lambda^{-} p_{0 i}^{-}, i=\overline{1,5}$, respectively will be equal: $\lambda_{01}^{+}=30, \quad \lambda_{02}^{+}=10, \quad \lambda_{03}^{+}=20, \quad \lambda_{04}^{+}=20, \quad \lambda_{05}^{+}=20$, $\lambda_{01}^{-}=15, \lambda_{02}^{-}=15, \lambda_{03}^{-}=15, \lambda_{04}^{-}=15, \lambda_{05}^{-}=15$. Suppose, that service rates of customers in QS are equal: $\mu_{1}=50, \mu_{2}=50, \mu_{3}=50, \mu_{4}=50, \mu_{5}=50$.

Let us also transition probabilities of positive and negative customers between QS be equal $p_{11}^{+}=0.1, \quad p_{12}^{+}=0.3, \quad p_{12}^{-}=0.1, \quad p_{1 i}^{+}=0.05, \quad p_{1 i}^{-}=0.05, \quad i=\overline{3,5}$, $p_{10}=1-\sum_{j=1}^{5}\left(p_{1 j}^{+}+p_{1 j}^{-}\right)=0.1 ; p_{21}^{+}=0.1, p_{21}^{-}=0.1, p_{22}^{+}=0.2, p_{22}^{-}=0.1, p_{2 i}^{-}=0.05$, $p_{2 i}^{+}=0.05, \quad i=\overline{3,5}, \quad p_{20}=1-\sum_{j=1}^{5}\left(p_{2 j}^{+}+p_{2 j}^{-}\right)=0.2, \quad p_{j i}^{-}=0.1, \quad p_{j i}^{+}=0.1, \quad i=\overline{1,3}$, $p_{j i}^{-}=0.05, p_{j i}^{+}=0.05, i=\overline{4,5}, j=\overline{3,5}, p_{i 0}=1-\sum_{j=1}^{5}\left(p_{i j}^{+}+p_{i j}^{-}\right)=0.2, i=\overline{3,5}$.

Calculations have shown that $a_{0}(t)=e^{-425 t}$.

Let's a random value $B_{i}$ has a Poisson distribution with parameter $\lambda>0$ and let $m=5$, then (1) takes form $\pi_{i m}=\frac{\lambda^{m}}{m !} e^{-\lambda}, m=\overline{0,5}, i=\overline{1,5}$. Let also $\lambda=5$.

We need to find, for example, state probability $P(1,2,3,4,5, t)$. It is the coefficient of $z_{1}^{1} z_{2}^{2} z_{3}^{3} z_{4}^{4} z_{5}^{5}$ in the expansion of $\Psi_{5}(z, t)$ in multiple series (12), so that the degree of $z_{i}$ must satisfy the relation $\alpha_{i}+l_{i}-q_{i}-m r_{i}-u_{i}-U-m w_{i}-m W=k_{i}$, $i=\overline{1,5}$, where $k_{1}=1, k_{2}=5, k_{3}=3, k_{4}=4, k_{5}=5$. Hence, it follows that

$$
\begin{gathered}
q_{i}=\alpha_{i}+l_{i}-m r_{i}-u_{i}-U-m w_{i}-m W-k_{i}, i=\overline{1,5}, \\
q_{i}=\alpha_{i}+l_{i}-m\left(r_{i}+w_{i}+\sum_{i=1}^{5} w_{i}\right)-\left(u_{i}+\sum_{i=1}^{5} u_{i}\right)-k_{i}, i=\overline{1,5}, \\
l_{i}+q_{i}+r_{i}+u_{i}+w_{i}=\alpha_{i}+2 l_{i}+r_{i}+u_{i}+w_{i}-m\left(r_{i}+w_{i}+\sum_{i=1}^{5} w_{i}\right)-\left(u_{i}+\sum_{i=1}^{5} u_{i}\right)-k_{i},
\end{gathered}
$$




$$
\begin{gathered}
l_{i}+q_{i}+r_{i}+u_{i}+w_{i}=\alpha_{i}+2 l_{i}+(1-m)\left(r_{i}+w_{i}\right)-m \sum_{i=1}^{5} w_{i}-\sum_{i=1}^{5} u_{i}-k_{i}, i=\overline{1,5}, \\
\sum_{i=1}^{5}\left(l_{i}+q_{i}+r_{i}+u_{i}+w_{i}\right)= \\
=\sum_{i=1}^{5}\left(\alpha_{i}+2 l_{i}\right)+5(1-m) \sum_{i=1}^{5}\left(r_{i}+w_{i}\right)-4\left(m W+U+k_{i}\right)
\end{gathered}
$$

Then from (12) we find that

$$
\begin{aligned}
& P(1,2,3,4,5, t)=e^{-425 t} \times
\end{aligned}
$$

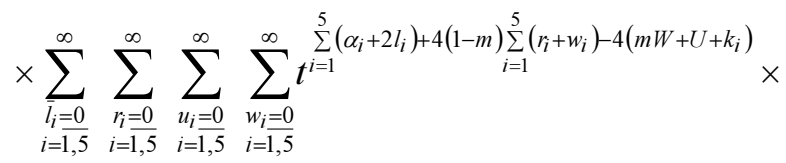

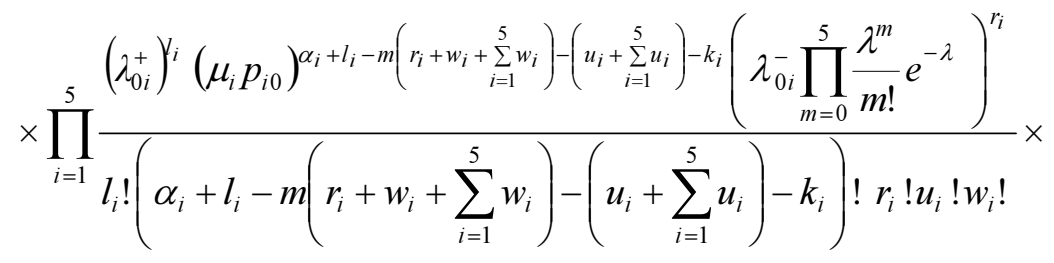

$$
\begin{aligned}
& \times \prod_{j=1}^{5}\left[\left(\mu_{j} p_{j i}^{+}\right)^{u_{j}}\left(\mu_{i} p_{4 i}^{+}\right)^{u_{4}}\left(\mu_{j} p_{j i}^{-} \prod_{m=0}^{5} \frac{\lambda^{m}}{m !} e^{-\lambda_{j}}\right)^{w_{j}}\right] .
\end{aligned}
$$

Figure 1 shows a chart of the state probability $P(1,2,3,4,5, t)$ for different $t$ on the condition that at the initial time, the network is in a state of $\alpha_{i}=0, i=\overline{1,5}$.

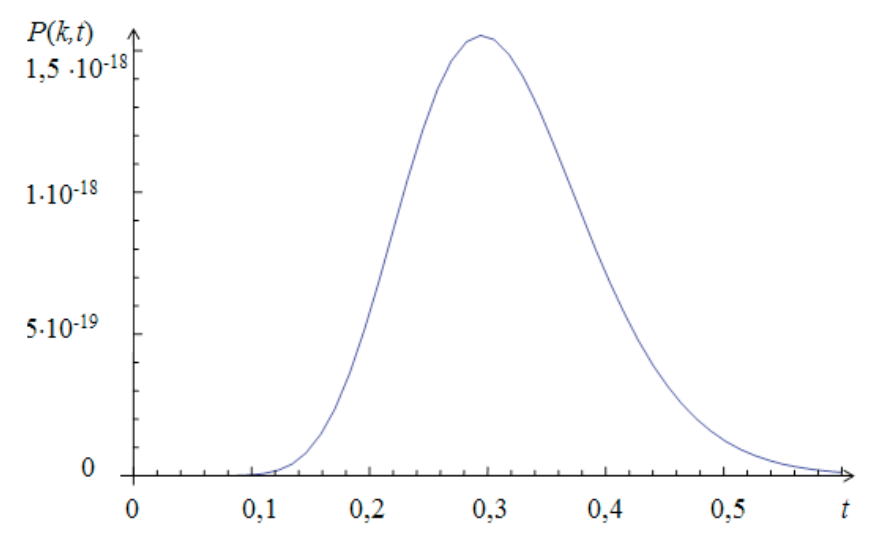

Fig. 1. The chart of the state probability $P(1,2,3,4,5, t)$ 
Calculating the partial derivative of the generating function (12) by variable $z_{i}$ in $\operatorname{dot} z=(1,1, \ldots, 1)$, and carrying out a series of mathematical transformations, we find that the mean number of customers in the queue system $S_{i}$ is calculated by the formula

$$
\begin{gathered}
N_{i}(t)=a_{0}(t) \sum_{k_{1}=0}^{\infty} \ldots \sum_{k_{i}=0}^{\infty} k_{i} \ldots \sum_{k_{n}=0}^{\infty} \ldots \sum_{r_{1}=0}^{\infty} \ldots \sum_{r_{n}=0}^{\infty} \sum_{u_{1}=0}^{\infty} \ldots \sum_{u_{n}=0}^{\infty} \sum_{w_{1}=0}^{\infty} \ldots \sum_{w_{n}=0}^{\infty} \\
\sum_{q_{1}=0}^{\alpha_{1}-m r_{1}-u_{1}-U-m w_{1}-m W} \ldots \sum_{q_{n}=0}^{\alpha_{n}-m r_{n}-u_{n}-U-m w_{n}-m W} \sum_{i=1}^{n}\left(k_{i}-\alpha_{i}+2 q_{i}+(m+1)\left(r_{i}+w_{i}\right)+2 u_{i}+U+m W\right) \\
\times \prod_{i=1}^{n} \frac{\left(\lambda_{0 i}^{+}\right)^{k_{i}-\alpha_{i}+q_{i}+m r_{i}+u_{i}+U+m w_{i}+m W}\left(\mu_{i} p_{i 0}\right)^{q_{i}}\left(\lambda_{0 i}^{-} \prod_{m=1}^{\infty} \pi_{i m}\right)^{n_{i}} \mu_{i}^{u_{i}+w_{i}}\left(\prod_{j=1}^{n} p_{i j}^{+}\right)^{u_{i}}\left(\prod_{j=1}^{n} \prod_{m=1}^{\infty} \pi_{i m} p_{i j}^{-}\right)^{w_{i}}}{\left(k_{i}-\alpha_{i}+q_{i}+m r_{i}+u_{i}+U+m w_{i}+m W\right) ! q_{i} ! r_{i} ! u_{i} ! w_{i} !} .
\end{gathered}
$$

Figure 2 shows a plot of changes of the mean number of customers in QS $S_{1}$.

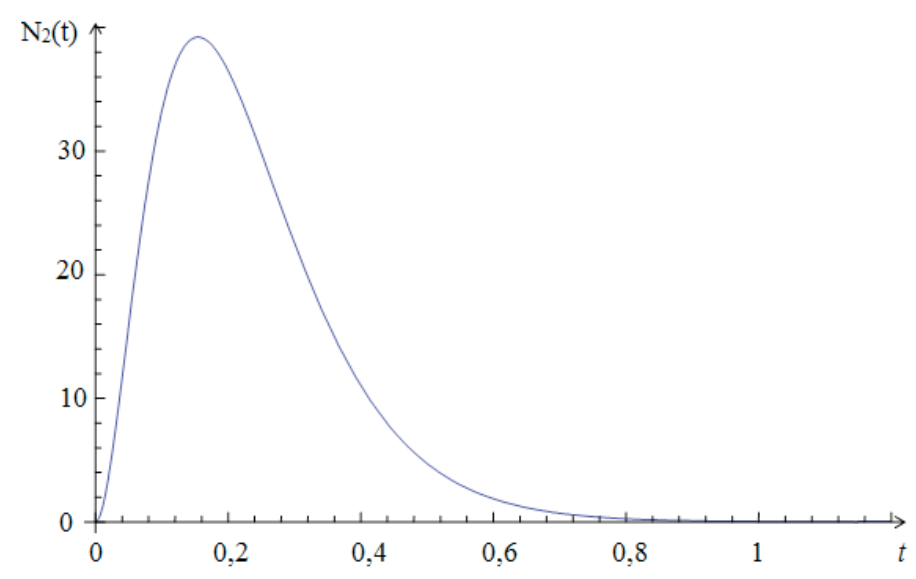

Fig. 2. The chat for changes of the mean number of positive customers $N_{2}(t)$ in QS $S_{2}$

\section{Conclusions}

In the article an investigation of Markov G-network was conducted in the case when a negative customer can destroy a random batch of positive customers. For such a network operating under a heavy traffic regime, finding a technique for non-stationary state probabilities and a mean number of customers in network systems was proposed using an apparatus based on the use of multivariate generating functions. 


\section{References}

[1] Gelenbe E., Product form queueing networks with negative and positive customers, Journal of Applied Probability 1991, 28, 656-663.

[2] Naumenko V., Pankov A., Kopats D., Investigation of Markov G-network with positive customers batch removal in transient regime. [In Russian: Issledovaniye markovskoy G-seti s gruppovym udaleniyem polozhitel'nykh zayavok v perekhodnom rezhime]. Vestnik of GrSU. Ser 2, 2016, 3 .

[3] Matalytski M., Naumenko V., Stochastic networks with non-standard customers movement. [In Russian: Stokhasticheskiye seti s nestandartnymi peremeshcheniyami zayavok], Monograph, GrSU, Grodno 2016.

[4] Naumenko V., Matalytski M., Network analysis with positive and negative requests in transitional mode. [In Russian: Analiz seti s polozhitel'nymi i otritsatel'nymi zaiavkami v perekhodnom rezhime], Vestnik of GrSU, Ser 2. 2016, 3(159), 135-142.

[5] Matalytski M., Naumenko V., Non-stationary analysis of queueing network with positive and negative messages, Journal of Applied Mathematics and Computational Mechanics 2013, 12(2), 61-71.

[6] Matalytski M., Naumenko V., Investigation of G-network with signals at transient behavior, Journal of Applied Mathematics and Computational Mechanics 2014, 13(1), 75-86.

[7] Matalytski M., Naumenko V., Investigation of G-network with random delay of signals at nonstationary behavior, Journal of Applied Mathematics and Computational Mechanics 2014, 13(3), 155-166.

[8] Gelenbe E., G-networks with signals and batch removal, Probability in the Engineering and Informational Sciences 1993, 7, 335-342. 